\title{
Berberine Improved Aldo-Induced Podocyte Injury via Inhibiting Oxidative Stress and Endoplasmic Reticulum Stress Pathways both In Vivo and In Vitro
}

\author{
Bin Wang ${ }^{a}$ Xianlin Xu Xiaozhou He $^{b}$ Zhigang Wang ${ }^{c}$ Min Yang ${ }^{a}$ \\ aDepartment of Nephrology, ${ }^{\mathrm{b} D e p a r t m e n t}$ of Urology, cDepartment of Respiration, the Third Affiliated \\ Hospital of Soochow University, Changzhou, China
}

\section{Key Words}

Berberine - Aldosterone - Podocyte - Oxidative stress - Endoplasmic reticulum stress Apoptosis

\begin{abstract}
Background/Aims: Berberine, a naturally occurring isoquinoline alkaloid, acts against oxidative stress (OS) and endoplasmic reticulum stress (ERS), both of which are responsible for Aldosterone (Aldo) -induced podocyte injury. However, the direct effects of berberine on Aldo-induced OS, ERS, and podocyte injury are not well defined. Methods: Uninephrectomized Sprague-Dawley rats were given $1 \% \mathrm{NaCl}$ (salt) in their water and an Aldo infusion $(0.75 \mu \mathrm{g} / \mathrm{h}$ ) for 28 days to induce podocyte injury in the Aldo group. In the Aldo/berberine group, in addition to Aldo infusion, rats were administered $150 \mathrm{mg} / \mathrm{kg}$ berberine per day by gastric gavage for 4 weeks. Podocytes were incubated in media containing either buffer or Aldo in the presence or absence of berberine for variable time periods. The kidney tissues and podocytes were then investigated using morphological analysis, immunohistochemistry, transmission electron microscopy, western blot, DHE staining, DCFDA fluorescence, and Annexin $V$ staining. Results: Here, we have reported that berberine attenuated Aldo-induced OS, ERS, and podocyte injury both in vivo and in vitro. Additionally, berberine treatment improved the extensive fusion of foot processes in electron micrographs resulting from Aldo/salt infusion in rats. Conclusion: Berberine may be examined as an effective agent against Aldo-induced podocyte injury.




\section{Cellular Physiology Cell Physiol Biochem 2016;39:217-228 and Biochemistry Published online: June 24, $2016 \quad \begin{aligned} & \text { DOI: 10.1159/000445618 } 2016 \text { The Author(s). Published by S. Karger AG, Basel } \\ & \text { www.karger.com/cpb }\end{aligned}$ \\ Wang et al.: Berberine Improved Aldo-Induced Podocyte Injury}

\section{Introduction}

The activation of the renin-angiotensin-aldosterone (Aldo) system (RAAS) plays a pivotal role in patients with hypertension [1], diabetes and other progressive nephropathies $[2,3]$ and may cause various physiological effects through both genomic and non-genomic mechanisms [4]. Although interruption of the RAAS by angiotensin-converting enzyme (ACE) inhibitors and angiotensin receptor blockers (ARBs) significantly reduces morbidity and mortality $[5,6]$ as well as the severity of proteinuria and nephrology in patients with chronic kidney disease (CKD) [7, 8], Aldo concentrations 'escape' to baseline during longterm therapy [9]. Recently, Aldo has become a focus of research as a dependent risk factor for CKD.

Podocytes are highly specialized cells that line the urinary side of the glomerular basement membrane. The lack of podocyte regeneration following cell injury or apoptosis is a major limitation in the approach to glomerular healing. In accordance with another research [10], we have demonstrated that both oxidative stress (OS) [11] and endoplasmic reticulum stress (ERS) [12] are involved in Aldo-induced podocyte injury. OS is characterized by the increased formation of reactive oxygen species (ROS), exceeding the endogenous antioxidant capacity, and DNA oxidative damage [13], whereas ERS is characterized by the increased expression of ERS markers, such as GRP78, CHOP, and so on [14]. Novel therapeutic strategies aimed at attenuating OS and/or ERS may ameliorate Aldo-induced podocyte injury.

Berberine is an isoquinoline alkaloid obtained from a number of important medicinal plant species $[15,16]$ and exhibits a variety of biochemical and pharmacological effects, such as antihypertensive [17], anti-inflammatory [18], and antidiabetic activities [19]. Although the low bioavailability [20] limited its wide use in clinical practice, berberine is already studied in a lot of clinical studies due to its various effects including lipid-lowering [21], changing the blood concentration of cyclosporin A in renal transplanted recipients [22], improving insulin resistance in type 2 diabetes mellitus patients [23, 24], and ameliorating inflammation in patients with acute coronary syndrome [25]. Several studies have reported that berberine exerts its beneficial effects in diabetic nephropathy (DN) through the suppression of OS and ERS $[26,27]$. As the activation of RAAS participates in kidney injury in human kidney tissues and experimental animal models of DN [28], the question arises whether berberine exerts its anti-OS and anti-ERS abilities in DN via directly attenuating hyperglycemia and/or indirectly suppressing excessive RAAS activation-induced OS and ERS. Furthermore, it remains unclear whether berberine directly inhibits RAAS-induced renal cell injury.

Here, we will focus on the direct effect of berberine on Aldo-induced podocyte injury. Hence, the aim of this study is to use in vivo and in vitro experiments to address whether berberine can protect podocytes against Aldo-induced injury and, if so, whether it exerts the effect through improving OS and ERS.

\section{Materials and Methods}

Antibodies and reagents

Aldo, berberine, and anti- $\beta$-actin antibody were purchased from Sigma (St. Louis, MO). Antibodies against CHOP and GRP78 were purchased from Cell Signaling Technology (Beverly, MA). Anti-podocin antibodies were obtained from Abcam (Cambridge, MA). All other chemicals were of analytical grade.

\section{Animals}

Study protocols were reviewed and approved by the Institutional Animal Care and Use Committee at Soochow University, China. In brief, 18 male Sprague-Dawley rats (5 - 6 weeks old, approximately 190 g) received a right uninephrectomy under light 3\% isoflurane anesthesia. Two weeks after the surgery, rats weighing 260 - 290 g were randomly divided into three groups for 4 weeks. In the Aldo group $(n=6)$, a subcutaneously osmotic minipump (model 2004; Alzet, Cupertino, CA) was implanted (at 0 weeks) under light $3 \%$ isoflurane anesthesia to infuse Aldo. In the Aldo/berberine group, in addition to the implantation of the minipump, rats were administered $150 \mathrm{mg} / \mathrm{kg}$ berberine per day by gastric gavage for 4 weeks. No 


\section{Cellular Physiology Cell Physiol Biochem 2016;39:217-228 \begin{tabular}{l|l} 
DOI: 10.1159/000445618 & Ond 2016 The Author(s). Published by S. Karger AG, Basel \\
www.karger.com/cpb
\end{tabular} \\ Wang et al.: Berberine Improved Aldo-Induced Podocyte Injury}

pump was implanted in the animals of the control group $(\mathrm{n}=6)$. Both groups received $1 \% \mathrm{NaCl}$ in their drinking water throughout the experimental period. Systolic blood pressure (SBP) was measured in the conscious state by tail-cuff plethysmography (BP-98A; Softron Co., Tokyo, Japan) at weeks 0 and 4 during the treatment period. Twenty-four-hour urine samples were collected starting after a 24-hour acclimatization period in metabolic cages. Urinary protein excretion was determined using enzyme-linked immunosorbent assay (ELISA) kits from Exocell (Philadelphia, PA, USA). Urine and plasma creatinine levels were analyzed using an assay kit (Jiancheng, Nanjing, China).

\section{Morphological analysis and immunohistochemistry}

Kidney sections ( $3 \mu \mathrm{m}$ thick) were stained with periodic acid-Schiff (PAS), and immunohistochemistry tests were performed according to an established procedure. The severity of glomerular injury was evaluated according to a previously described method $[29,30]$. In brief, the severity of injury for each glomerulus was scored from 0 to 4: 0 , no lesion; $1,<25 \%$ involvement of the glomerulus; 2,25 - 50\% involvement; 3, 50 - 75\% involvement; and 4, > 75\% involvement. Fifty glomeruli were analyzed per kidney section. A glomerular sclerosis score (GSS) for each animal was calculated by multiplying each severity score (0$4+$ ) by the percentage of glomeruli displaying that degree of injury and summing these scores. The above histological analysis was performed in a blinded manner to avoid bias.

Immunohistochemical staining was performed on formalin-fixed, paraffin-embedded $3 \mu \mathrm{m}$ sections, which were rehydrated, and the antigens were retrieved using heated citrate. The sections were incubated with goat anti-podocin antibody $(1: 200)$ overnight at $4^{\circ} \mathrm{C}$, and the staining was visualized using horseradishperoxidase coupled secondary antibodies (Vectastain Elite, Vector Labs). Related isotype immunoglobulins (Jackson Immuno Research, USA) were used as the negative controls. Integrated optical density (IOD) was used to represent the relative amount of positive staining, and twenty randomly chosen glomeruli were analyzed at a magnification $\times 400$. All immunohistochemical analyses were repeated at least three times, and representative images were presented. All analyses were performed in a masked manner.

\section{DHE Staining in Kidney Sections and Urinary 8-hydroxy-2'-deoxyguanosine (8-OHdG) excretion}

Frozen kidney segments in OCT compound were cut into $3 \mu \mathrm{m}$ sections and stained with DHE $(50 \mu \mathrm{M}$, Invitrogen, Carlsbad, CA) for exactly 30 min at room temperature in the dark. Images were taken using a laser scanning confocal microscope system (Bio-Rad Laboratories, Hercules, CA). DHE fluorescent images were visualized by excitation at $488 \mathrm{~nm}$ and emission at $610 \mathrm{~nm}$ to detect the oxidized DHE product ethidium. We evaluated the density using Image pro plus 6.0 software. We used "irregular AOI" to choose the glomerulus and measured the intensity and the relative fluorescence values were corrected by the number of cells in each glomerulus. The average DHE fluorescence intensities of the glomerulus were calculated using at least 20 glomeruli from each sample.

Urinary 8-hydroxy-2'-deoxyguanosine (8-OHdG) excretion was determined using commercially available kits (Nikken Seil, Shizuoka, Japan, and microTP-test, Wako, Osaka, Japan).

\section{Glomerular isolation}

Glomeruli were isolated as previously reported [31]. The purity of the glomerular preparation was $>95 \%$ as determined by light microscopy. Briefly, kidneys were first perfused with a mixture of Dynabeads (diameter $4.5 \mu \mathrm{m}$ ) and iron powder (diameter $6 \mu \mathrm{m}$ ). The tissues were then rinsed on a $70 \mu \mathrm{m}$ nylon mesh after magnetic treatment to remove small tubular fragments.

\section{Transmission electron microscopy}

Kidney sections ( $3 \mu \mathrm{m}$ thick) were stained with periodic acid-Schiff (PAS), and immunohistochemistry was performed according to an established procedure [13]. Slit pore diameter was measured as previously described [30,32].

\section{Podocyte culture and treatment}

The conditionally immortalized mouse podocyte cell line MPC5 was cultured as previously described [11]. Podocytes were maintained without interferon $\gamma$ at $37^{\circ} \mathrm{C}$ for 14 days before experimentation to induce differentiation. Differentiated podocytes were made quiescent in medium that contained $0.1 \%$ FBS for $24 \mathrm{~h}$, and the cells were then exposed to treatment for the indicated time periods. 


\section{Cellular Physiology Cell Physiol Biochem 2016;39:217-228

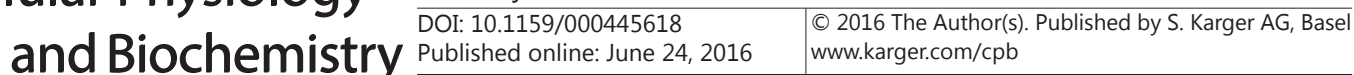 \\ Wang et al.: Berberine Improved Aldo-Induced Podocyte Injury}

The LDH toxicity assay

Cultured cells were treated with different concentrations $(5,10,25,50,75$, and $100 \mu \mathrm{M})$ of berberine or without berberine for $48 \mathrm{~h}$, and the LDH activity was assayed by the absorbance change at a wavelength of $440 \mathrm{~nm}$ using an LDH assay kit (Jiancheng, Nanjing, China).

Hoechst 33258 staining

Podocytes grown on glass cover slips in the different groups were stained with Hoechst 33258 and viewed by fluorescence microscopy to measure apoptosis.

Annexin V-fluorescein isothiocyanate conjugated with propidium iodide staining

After treatment, podocytes of different groups were quantified by Annexin $V$ staining according to the manufacturer's instructions (BD Biosciences, San Diego, CA). After blowing the cell suspension evenly, we placed a drop on glass slides and observed the cells by fluorescence microscopy. The remaining cells were used for the measurement of apoptosis on a FACScan flow cytometer (Epics Altra, Beckman Coulter, Brea, $\mathrm{CA})$.

ROS

Podocytes were seeded into six well plates, and allowed to grow until they reached confluent cell density. Plates were treated with $10 \mu \mathrm{l}$ DCFDA for $30 \mathrm{~min}$ at $37^{\circ} \mathrm{C}$ in the dark. Podocytes were then washed twice, and fluorescence was measured using a fluorescence plate reader at the excitation and emission wavelengths of 485 and $535 \mathrm{~nm}$, respectively.

Western blot analysis

Podocytes harvested from plates and sieved glomeruli were lysed in SDS sample buffer containing $150 \mathrm{mM} \mathrm{NaCl}, 0.1 \%$ Triton X-100, $0.5 \%$ deoxycholate, $0.1 \%$ sodium dodecyl sulfate (SDS), $50 \mathrm{mM}$ Tris-HCl (pH 7.0), and $1 \mathrm{mM}$ ethylenediaminetetraacetic acid (EDTA). Protein expression was detected by Western blot according to established protocols. The primary antibodies used were as follows: GRP78 (1:1000), CHOP (1:1000), podocin (1:1000), and $\beta$-actin (1:10000). Densitometric analysis was performed using the Quantity One Software (Bio-Rad). The relative intensity of each band was normalized to the band of $\beta$-actin.

Statistical Analyses

Data are expressed as the mean \pm standard error of mean (SEM). Comparisons between groups were performed using one-way ANOVA followed by Dunnett's multiple comparison tests or Student's t-test. $\mathrm{P}<0.05$ was considered statistically significant.

\section{Results}

\section{Physiological parameters}

As shown in Table 1, the Aldo/salts group showed an increased urinary Albumin/ creatinine ratio compared with the control group $(\mathrm{P}<0.05)$ and was improved by berberine treatment $(\mathrm{P}<0.05)$. In addition, the Aldo group showed significant increases in the kidney/ body weight ratio and urine volume; however, berberine had no additional effect on these two parameters. Urinary Aldo levels were higher in the Aldo group and the Aldo plus berberine group, verifying correct pump function. Compared to the control group, Aldo/salt treatment resulted in marginally higher SBP over time (final measurement, $188 \pm 5 \mathrm{mmHg}$ and $140 \pm 3$ $\mathrm{mmHg}$, respectively), and the administration of berberine had no effect on SBP compared to Aldo-treated rats (final measurement, $177 \pm 6$ and $188 \pm 5 \mathrm{mmHg}$, respectively). There was no significant difference in serum creatinine levels among all groups.

Berberine treatment ameliorated Aldo-induced histologic injury in rats

PAS staining and semi-quantification revealed marked Aldo-induced glomerular enlargement and increased mesangial area and resulted in increased glomerular sclerosis scores compared with the control group (Fig. 1A and B). In contrast, the berberine groups 


\section{\begin{tabular}{lll} 
Cellular Physiology & \multicolumn{1}{c}{ Cell Physiol Biochem 2016;39:217-228 } \\
DOI: 10.1159/000445618 & O 2016 The Author(s). Published by S. Karger AG, Basel
\end{tabular}

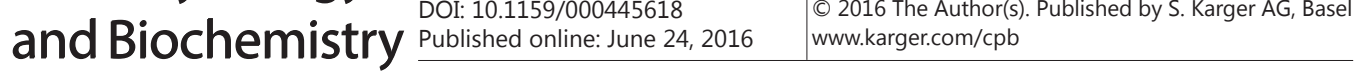

Table 1. Biological parameters of rats in the control, Aldo, and Aldo/berberine groups at 4 weeks. ${ }^{*} \mathrm{P}<0.05$ vs Control group, \# $\mathrm{P}<0.05$ vs Aldo group

Fig. 1. Histological findings on glomeruli from different groups of rats. (A) The light microscopic appearance of representative glomeruli is shown by PAS staining ( $\times 400)$. (B) Results of the semi-quantitative analysis. The data are expressed as the mean $\pm \mathrm{SEM} ; \mathrm{n}=6$ per group. ${ }^{\#} \mathrm{P}<0.05$ vs control group, and * $\mathrm{P}$ $<0.05$ vs Aldo group.

\begin{tabular}{llll}
\hline & Control & Aldo & Aldo/berberine \\
\hline Body weight (g) & $464 \pm 11$ & $452 \pm 9$ & $405 \pm 7^{*}$ \\
Kidney weight/body weight ratio (mg/g) & $5.8 \pm 0.2$ & $9.5 \pm 0.6^{*}$ & $9.1 \pm 0.6^{*}$ \\
Urine volume (ml) & $12 \pm 3$ & $45 \pm 11^{*}$ & $33 \pm 5^{*}, \#$ \\
Creatinine clearance (ml/min) & $4.1 \pm 0.4$ & $2.9 \pm 0.2$ & $4.5 \pm 0.6$ \\
Albumin/creatinine (mg/mg) & $1.4 \pm 0.5$ & $9.3 \pm 2.4^{*}$ & $5.7 \pm 1.4^{*}, \#$ \\
Urinary aldosterone at end ( $\mu \mathrm{g} / 24 \mathrm{~h})$ & $0.04 \pm 0.01$ & $0.15 \pm 0.03^{*}$ & $0.14 \pm 0.01^{*}$ \\
SBP $(\mathrm{mmHg})$ & $140 \pm 3$ & $188 \pm 5^{*}$ & $177 \pm 6^{*}$ \\
\hline
\end{tabular}

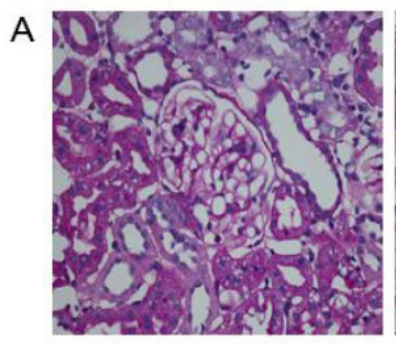

Control

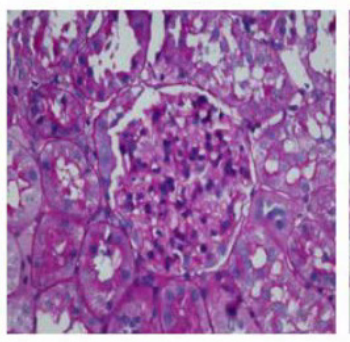

Aldo

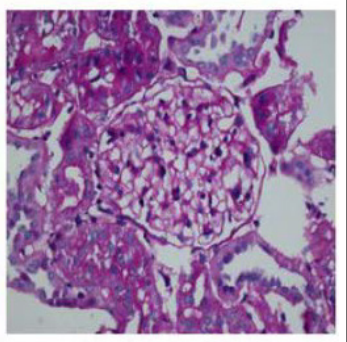

Aldo+Berberine

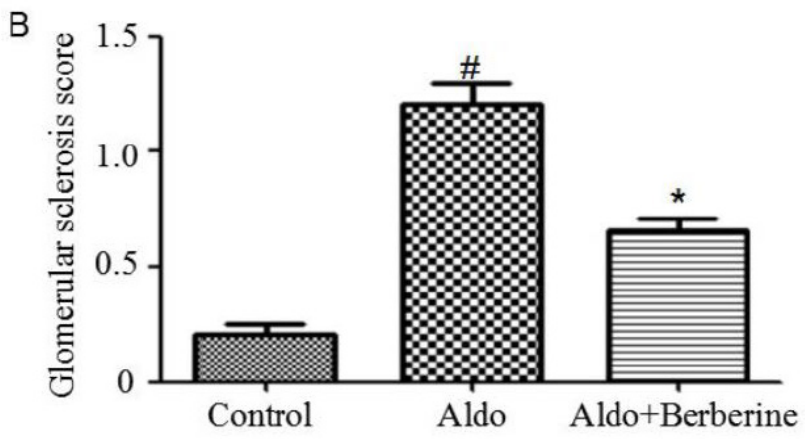

showed an improved histological appearance (Fig. 1A) and significantly lower Aldo-induced increase in glomerular injury scores (Fig. 1B).

\section{Berberine treatment ameliorated Aldo-induced podocyte injury in rats}

Having proven that Aldo contributes to podocyte injury, which is responsible for glomerular sclerosis [11], we then examined whether berberine could protect podocytes against Aldo-induced injury. As shown in Fig. 2A and B, the Aldo group showed reduced expression of the glomerular slit diaphragm protein podocin and extensive fusion of foot processes in electron micrographs compared with the control group. However, berberine treatment ameliorated all the changes mentioned above (Fig. 2C and D).

\section{Berberine treatment ameliorated Aldo-induced OS in rats}

As OS is among the major pathways that contribute to Aldo-induced podocyte injury [11], the ROS level in the glomeruli and urinary 8-OHdG excretion were evaluated. Both urinary 8-OHdG excretion and DHE fluorescence in the glomeruli were markedly increased in the Aldo group (Fig. 3A and B). As expected, both ROS formation and 8-OHdG level were reduced in the Aldo plus berberine group compared with the Aldo group (Fig. 3A and B).

Berberine treatment ameliorated Aldo-induced ERS in rats

As we have previously demonstrated that ERS is also involved in Aldo-induced podocyte injury, we reasoned that attenuating ERS might also contribute to the protective effect of 
Fig. 2. Effects of berberine treatment on Aldo-induced podocyte injury in rats of different groups. (A) Immunohistochemical staining of podocin in kidney sections from different groups. (B) These areas were also assessed quantitatively, as described in Materials and Methods. (C) Foot processes of podocytes observed by transmission electron microscopy (TEM) $(\times 4,200)$. (D) Quantitative analysis of the slit pore diameter. The data are expressed as the mean \pm SEM; $\mathrm{n}=6$ per group. ${ }^{*} \mathrm{P}<0.05$ vs. control group, and ${ }^{*} \mathrm{P}<0.05$ vs. Aldo group.

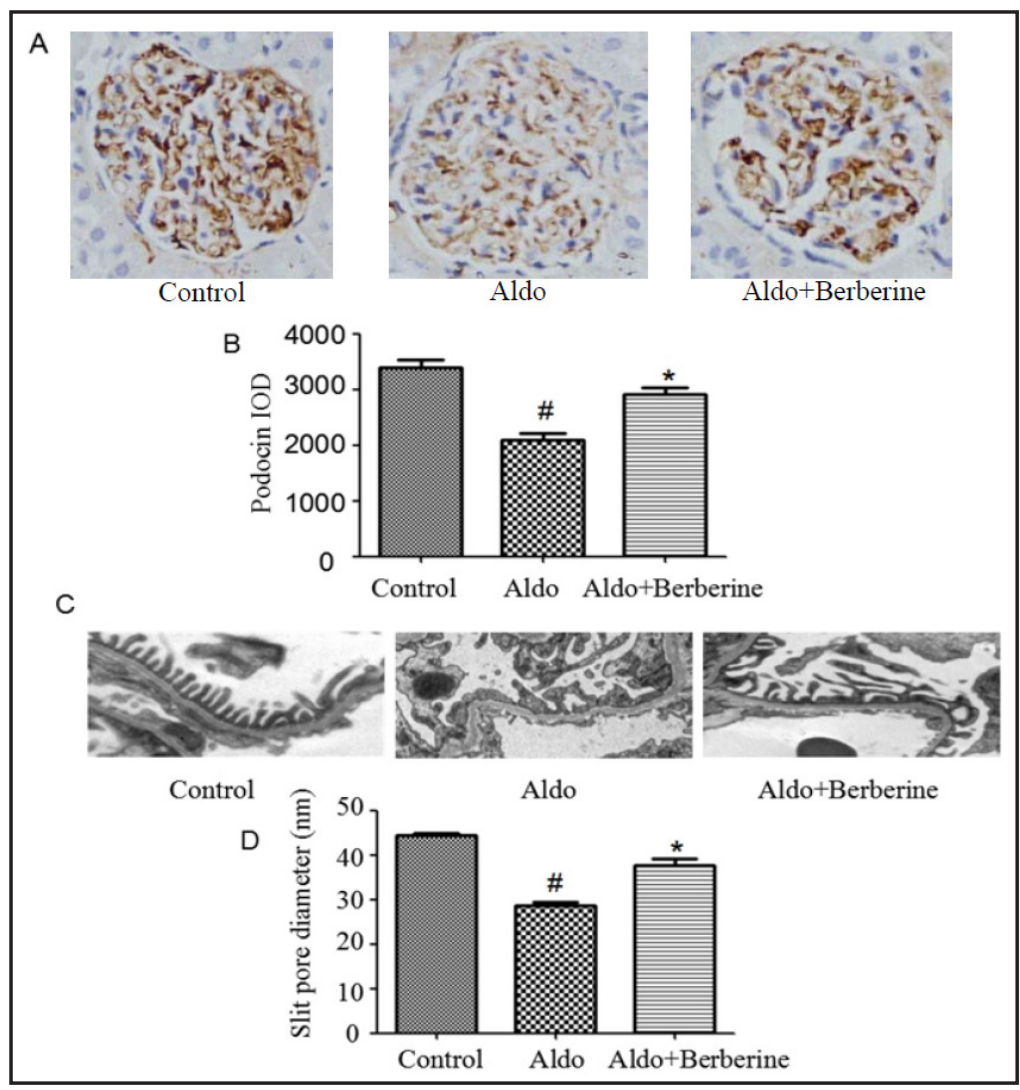

Fig. 3. Effects of berberine treatment on oxidative stress markers evaluated based on urinary 8-OHdG excretion (A) and dihydroethidium (DHE) staining (B) in the glomeruli of rats in the respective groups. The data are expressed as the mean \pm SEM; $\mathrm{n}=6$ per group. ${ }^{\#} \mathrm{P}<0.05$ vs control group, and ${ }^{*} \mathrm{P}<0.05$ vs Aldo group.

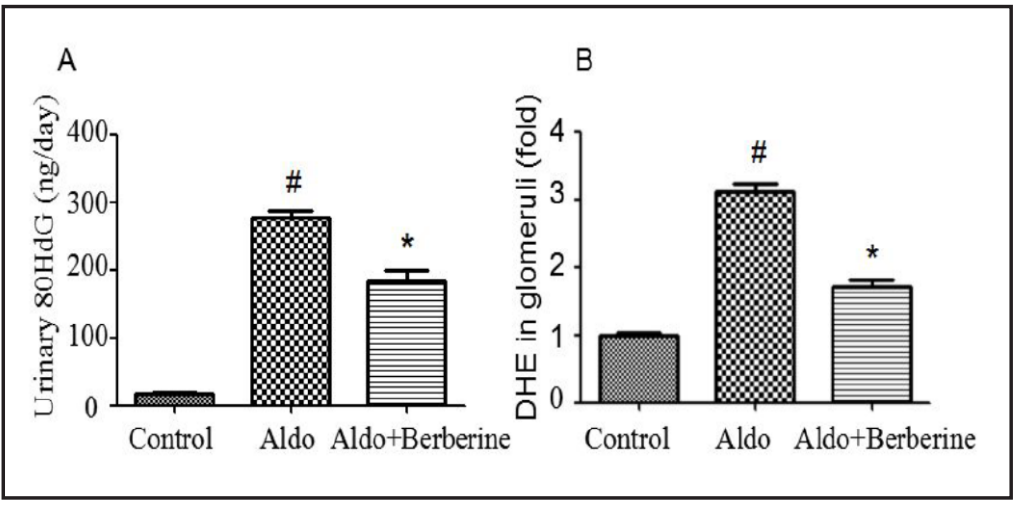

Fig. 4. Effects of berberine treatment on Aldo-induced ERS in the glomeruli of rats (A) Western blot analysis of the whole cell lysate, immunoblotted with GRP78 and CHOP antibodies. (B) Quantitative ratios are shown as relative optical densities of bands after normalization to $\beta$-actin expression. The data are representative of three similar experiments and are quantified as the mean \pm SEM. ${ }^{*} \mathrm{P}<0.05$ vs control group, and ${ }^{*} \mathrm{P}<0.05$ vs Aldo group.

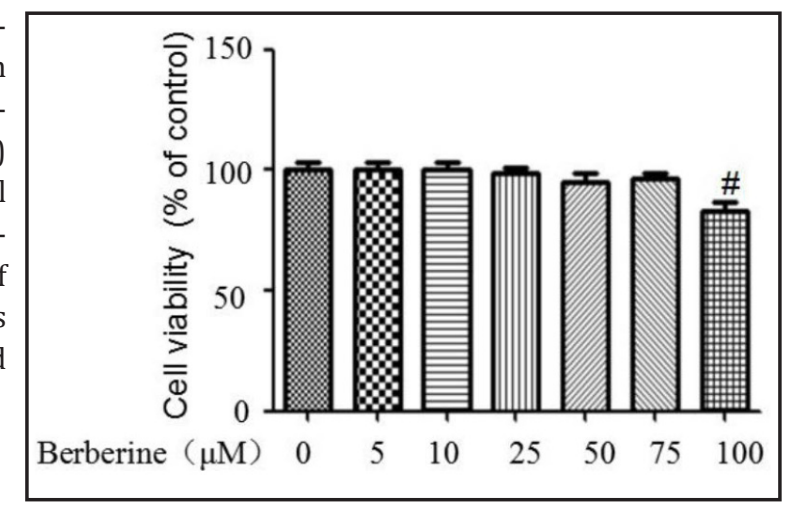

berberine against Aldo-induced glomerular injury. Aldo-induced ERS, indicated by the increase in ERS markers GRP78 and CHOP in comparison with the rats of the control group, was attenuated by berberine treatment (Fig. 4A and B).

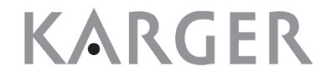




\section{Cellular Physiology Cell Physiol Biochem 2016;39:217-228 and Biochemistry \begin{tabular}{l|l} 
DOI: 10.1159/000445618 & (c) 2016 The Author(s). Published by S. Karger AG, Base \\
www.karger.com/cpb
\end{tabular}

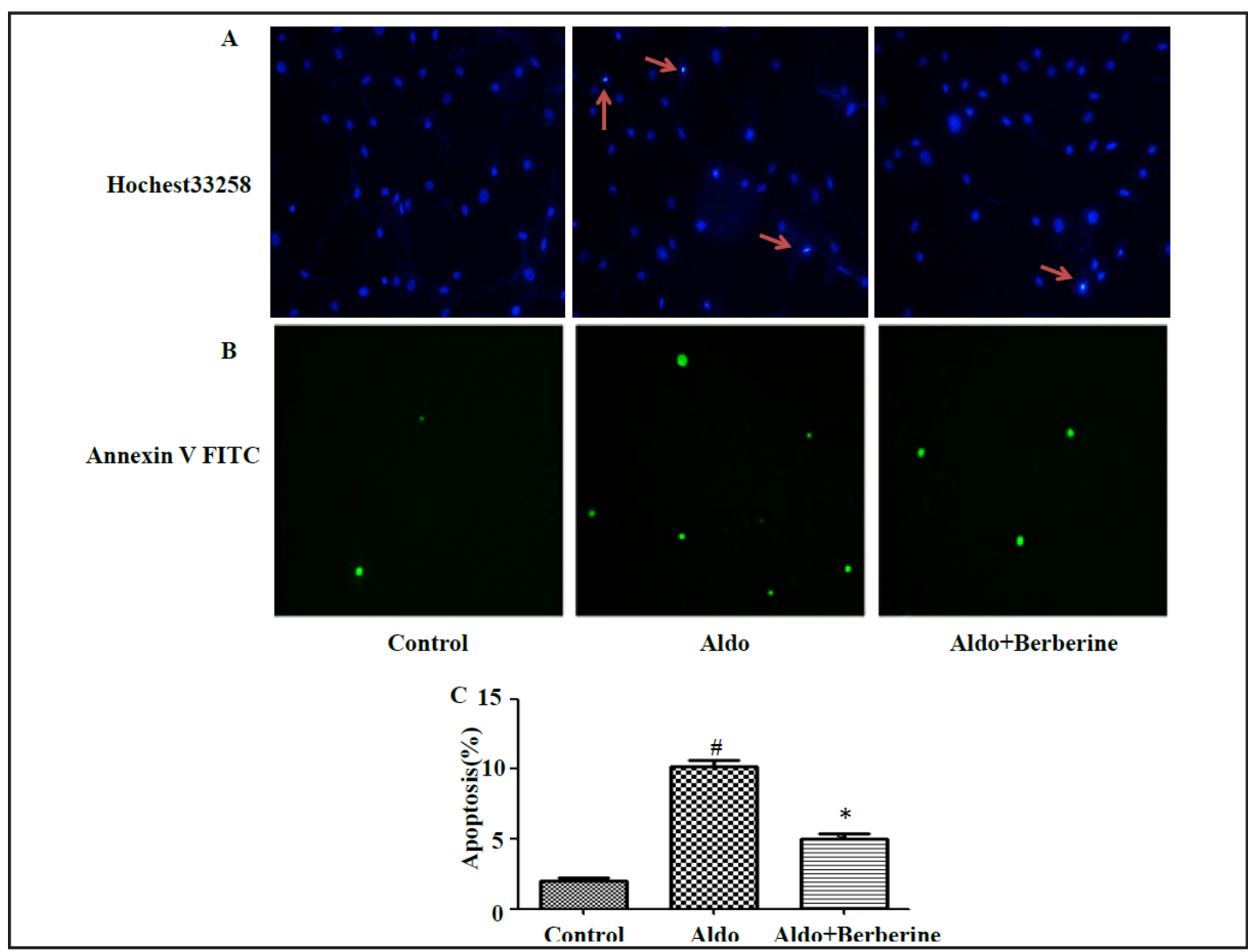

Fig. 5. The cytotoxic effects of berberine on podocytes. The LDH toxicity assay, as described in Materials and Methods, is used to measure the toxic effects of different concentrations of berberine $(0,5,10,25,50,75$, and $100 \mu \mathrm{M}$ ) and is expressed in terms of the percentage of dead cells. The values are given as the mean \pm S.D. from 6 independent experiments. The absorbance readings obtained by cracking all cells in the control group was arbitrarily defined as $100 \%$ cytotoxicity. ${ }^{\#} \mathrm{P}<0.05$ vs Control group.

\section{Berberine exerted limited effect on podocytes viability}

To extend our in vivo results to the in vitro condition, cultured mouse podocytes were used to evaluate the protective effects of berberine on Aldo-induced injury. First, we need to determine which intervention concentration is the best one. As shown in Fig. 5, under normoxic conditions, treating podocytes with doses of berberine below $75 \mu \mathrm{M}$ for $48 \mathrm{~h}$ caused little effect on cell viability; however, $100 \mu \mathrm{M}$ berberine significantly enhanced cytotoxicity $(\mathrm{P}<0.05)$. Thus, we chose the condition of $75 \mu \mathrm{M}$ for $48 \mathrm{~h}$ as one of the most frequent procedures performed in our vitro experiments.

\section{Berberine protected against Aldo-induced podocyte apoptosis in vitro}

Both annexin V/flow cytometry detection and Hoechst 33258 staining were applied to label the apoptosis of podocytes. In accordance with the protective effect of berberine against Aldo-induced podocyte injury in vivo, berberine pretreatment reduced Aldo-induced podocyte apoptosis in vitro (Fig. 6A through C).

Berberine attenuated Aldo-induced podocyte injury, OS, and ERS in vitro

It is known that both OS and ERS contribute to Aldo-induced podocyte injury. The ROS level and the expression of GRP78 and CHOP were evaluated to investigate whether berberine protects podocytes against Aldo-induced injury via inhibiting OS and/or ERS in vitro. As shown in Fig. 7A through D, the ROS level and the protein levels of GRP78 and CHOP in the Aldo group were upregulated compared with control group. After berberine treatment, the ROS level and the expression of GRP78 and CHOP decreased. Furthermore, 


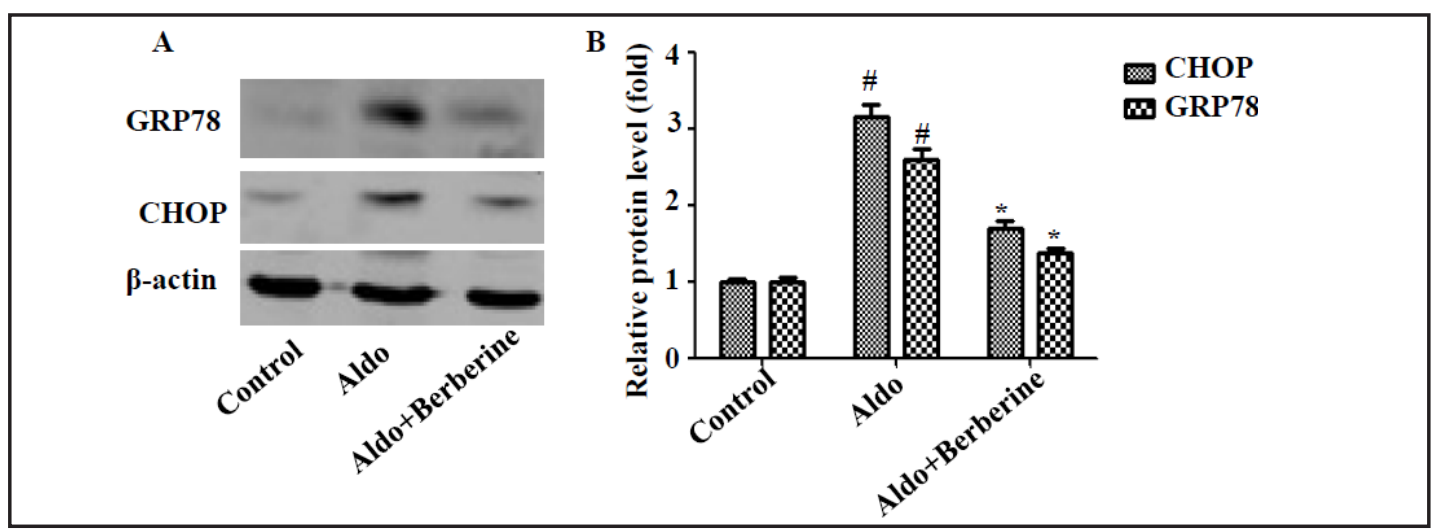

Fig. 6. Effect of berberine on Aldo-induced podocyte apoptosis. (A) Hoechst 33258 staining in podocytes after various treatments, as indicated. The red arrow indicates apoptosis-induced chromatin condensation and fragmentation. Magnification is $\times 200$. (B)Annexin V-FITC labeled apoptotic podocytes viewed under the fluorescence microscope. Magnification is $\times 200$. (C) Quantification of apoptotic cells by flow cytometry. The results (mean $\pm \mathrm{SEM}$ ) of 3 series of experiments. ${ }^{*} \mathrm{P}<0.05$ vs control group, and ${ }^{*} \mathrm{P}<0.05$ vs Aldo group.

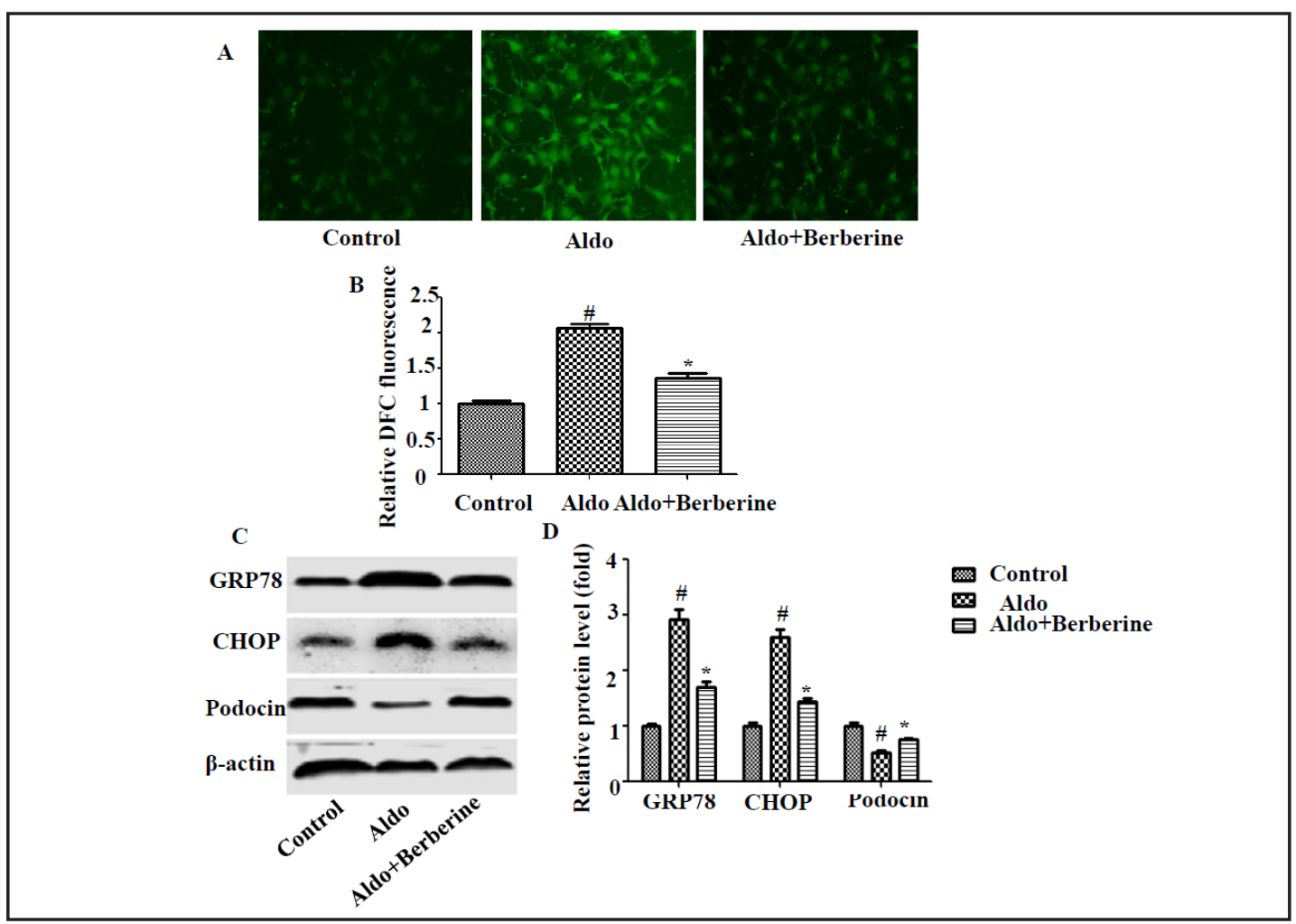

Fig. 7. Effects of berberine on Aldo-induced increase in ROS in cultured podocytes. (A) Representative images of podocytes stained with dichlorodihydrofluorescein diacetate. (B) Quantification of 2', 7'-dichlorofluorescein (DCF) fluorescence. (C) Western blots analysis of the whole cell lysate, immunoblotted with GRP78, CHOP and podocin antibodies. (D) Quantitative ratios are shown as the relative optical densities of bands after normalization to $\beta$-actin expression. The data are representative of three similar experiments and are quantified as the mean \pm SEM. ${ }^{*} \mathrm{P}<0.05$ vs control group, and ${ }^{*} \mathrm{P}<0.05$ vs Aldo group.

pretreatment with berberine inhibited the reduction of the glomerular slit diaphragm protein podocin induced by Aldo (Fig. 7C and D). These results indicate that berberine could attenuate podocyte injury through improving OS and ERS. 


\section{Cellular Physiology Cell Physiol Biochem 2016;39:217-228 \\ \begin{tabular}{l|l} 
and Biochemistry Published online: June 24, 2016 & $\begin{array}{l}\text { (c) 2016 The Author(s). Published by S. Karger AG, Basel } \\
\text { www.karger.com/cpb }\end{array}$
\end{tabular} \\ Wang et al.: Berberine Improved Aldo-Induced Podocyte Injury}

\section{Discussion}

In this study, our results suggest that both OS and ERS participate in Aldo-induced podocyte injury; however, berberine can attenuate all the changes both in vivo and in vitro, indicating the potential of berberine as a new strategy to ameliorate Aldo-induced podocyte injury.

The activation of the RAAS system is a major hallmarkin the development and progression of organ damage in CKD, and Aldo concentrations are inappropriately high in many patients with CKD or hypertension, as well as in an increasing number of individuals with metabolic syndrome and sleep apnea [33]. Growing evidence suggests that Aldo induces OS, ERS, podocyte injury, and subsequent glomerular sclerosis $[10,11]$. MR antagonism improves kidney injury in patients with CKD and in many animal models of progressive nephropathies [34]. Consistently with previous studies $[10,11]$, Aldo contributes to podocyte injury in vivo, as indicated by the increases in the urinary protein/creatinine ratio (Table 1), glomerular sclerosis (Fig. 1), the reduced expression of the glomerular slit diaphragm protein podocin (Fig. 7), and extensive fusion of foot processes in electron micrographs (Fig. 2). The results in vitro study also support Aldo-induced podocyte injury, as evidenced by increased apoptosis and the reduced expression of podocin (Fig. 6 and 7).

Natural products and their synthetic derivatives have been a continuous source of novel compounds for the treatment of various diseases [35-37]. In this investigation, we showed that a naturally occurring isoquinoline alkaloid, berberine, significantly attenuated all the Aldo-induced changes mentioned above (Table 1, Fig. 1, 2, 6 and 7). Although, our data still demonstrated that berberine had some effect on body weight loss, maybe due to its lipid-lowering effects [38] and inhibition of food and water intake [39]. Importantly, we found that berberine was not toxic to podocytes under the conditions used, except for a moderate reduction in cell viability at higher concentrations (Fig. 5). These results suggest that berberine may be a safe and effective agent for curing Aldo-induced podocyte injury. However, the mechanisms involved in the protective effect of berberine on Aldo-induced podocyte injury remain far from clear.

It has been acknowledged that Aldo-induced ROS production occurs via NADPH oxidase, and OS plays a leading role in the pathogenesis of Aldo-induced kidney injury [40, 41]. Scavenger receptor (SR) is believed to be exerting an anti-OS effect via increasing the clearance of oxidized lipoproteins and the resolution of inflammatory processes, changing cellular functions such as gene expression and signaling [42]. Although we did not detect the SR expression in our experiments, a previous study has proven that berberine could induce SR-A expression in macrophages [43]. Additionally, berberine could scavenge the reactive oxygen species (ROS) and inhibit the c-jun NH (2)-terminal kinase (JNK), the loss of mitochondrial membrane potential and the release of cytochrome c (Cyt C) and caspase- 3 [44]. In our study, Aldo-treated rats showed a high glomerular ROS production marker (Fig. 3B) and a significant increase in urinary 8-OHdG excretion, a marker of systemic OS (Fig. 3A). Berberine treatment reduced the OS induced by Aldo both in vivo (Fig. 3) and in vitro (Fig. 7). Hence, our results indicated that berberine attenuated Aldo-induced podocyte injury at least partially via suppressing OS.

Under ER stress conditions, an imbalance between protein-folding capacity and protein-folding load leads to the induction of the unfolded protein response (UPR), which serves as an adaptive response. However, if ERS is severe or the duration of ERS is too long, it also induces cell apoptosis, and a large amount of data suggests that CHOP is a crucial component of the ERS-induced cell death pathway $[45,46]$. Dysfunctions of the endoplasmic reticulum (ER) in maintaining protein homeostasis can result from OS [14, 47]. Here, we found that the expression levels of the ERS-associated proteins GRP78 and CHOP were up-regulated following Aldo stimulation both in vivo and in vitro, which was significantly inhibited by treatment with berberine (Fig. 3, 4 and 7). The results suggest that berberine may also protect podocytes against Aldo-induced injury partially through attenuating overt ERS. Oxidative stress induces ER stress through the accumulation of reactive oxygen species 


\section{Cellular Physiology Cell Physiol Biochem 2016;39:217-228 \begin{tabular}{c|c|c|} 
DOI: 10.1159/000445618 & $\begin{array}{l}\text { O) 2016 The Author(s). Published by S. Karger AG, Basel } \\
\text { www.karger.com/cpb }\end{array}$ \\
\hline and Biochemistry & Published online: June 24, 2016
\end{tabular} \\ Wang et al.: Berberine Improved Aldo-Induced Podocyte Injury}

and vice versa. Hence, it is easy to be understood that berberine exerted a role of both antiOS and anti-ERS effects in our model.

In conclusion, our study provides the first evidence that berberine may be examined as an effective agent against RAAS activation-induced podocyte injury through suppressing both OS and ERS. Hence, berberine indirectly suppresses excessive RAAS activation-induced OS and ERS may also participate in berberine's anti-OS and anti-ERS effects in DN.

\section{Acknowledgments}

This work was supported by grants from applied basic research project of Changzhou Municipal Science and Technology Bureau, the project for training high-level health personnel in Changzhou City and the Health Bureau major projects of Changzhou City (NO.ZD201402).

\section{Disclosure Statement}

No conflict of interest.

\section{References}

1 Jugdutt BI: Expanding Saga of the Renin-Angiotensin System: The Angiotensin II Counter-Regulatory AT2 Receptor Pathway. Circulation 2015;131:1380-1383.

2 Mavrakanas TA, Gariani K, Martin PY: Mineralocorticoid receptor blockade in addition to angiotensin converting enzyme inhibitor or angiotensin II receptor blocker treatment: an emerging paradigm in diabetic nephropathy: a systematic review. Eur J Intern Med 2014;25:173-176.

3 Ruster C, Wolf G: Renin-angiotensin-aldosterone system and progression of renal disease. J Am Soc Nephrol 2006;17:2985-2991.

4 Boldyreff B, Wehling M: Non-genomic actions of aldosterone: mechanisms and consequences in kidney cells. Nephrol Dial Transplant 2003;18:1693-1695.

5 Bomback AS, Kshirsagar AV, Amamoo MA, Klemmer PJ: Change in proteinuria after adding aldosterone blockers to ACE inhibitors or angiotensin receptor blockers in CKD: a systematic review. Am J Kidney Dis 2008;51:199-211.

6 Toto R, Palmer BF: Rationale for combination angiotensin receptor blocker and angiotensin-converting enzyme inhibitor treatment and end-organ protection in patients with chronic kidney disease. Am J Nephrol 2008;28:372-380.

7 Ruggenenti P, Cravedi P, Remuzzi G: Mechanisms and treatment of CKD. J Am Soc Nephrol 2012;23:19171928.

8 Strippoli GF, Craig MC, Schena FP, Craig JC: Role of blood pressure targets and specific antihypertensive agents used to prevent diabetic nephropathy and delay its progression. J Am Soc Nephrol 2016;17:S153-S155.

9 Schrier RW, Masoumi A, Elhassan E: Aldosterone: role in edematous disorders, hypertension, chronic renal failure, and metabolic syndrome. Clin J Am Soc Nephrol 2010;5:1132-1140.

10 Yuan Y, Huang S, Wang W, Wang Y, Zhang P, Zhu C, Ding G, Liu B, Yang T, Zhang A: Activation of peroxisome proliferator-activated receptor-gamma coactivator 1alpha ameliorates mitochondrial dysfunction and protects podocytes from aldosterone-induced injury. Kidney Int 2012;82:771-789.

11 Yang M, Wang B, Li M, Jiang B: Connexin 43 is involved in aldosterone-induced podocyte injury. Cell Physiol Biochem 2014;34:1652-1662.

12 Yuan Y, Xu X, Zhao C, Zhao M, Wang H, Zhang B, Wang N, Mao H, Zhang A, Xing C: The roles of oxidative stress, endoplasmic reticulum stress, and autophagy in aldosterone/mineralocorticoid receptor-induced podocyte injury. Lab Invest 2015;95:1374-1386.

13 Queisser N, Amann K, Hey V, Habib SL, Schupp N: Blood pressure has only minor influence on aldosteroneinduced oxidative stress and DNA damage in vivo. Free Radic Biol Med 2013;54:17-25. 


\section{Cellular Physiology Cell Physiol Biochem 2016;39:217-228 \begin{tabular}{ll|l} 
and Biochemistry & Dublished online: June 24, 2016 & $\begin{array}{l}\text { C } 2016 \text { The Author(s). Published by S. Karger AG, Basel } \\
\text { www.karger.com/cpb }\end{array}$
\end{tabular} \\ Wang et al.: Berberine Improved Aldo-Induced Podocyte Injury}

14 Ding W, Yang L, Zhang M, Gu Y: Reactive oxygen species-mediated endoplasmic reticulum stress contributes to aldosterone-induced apoptosis in tubular epithelial cells. Biochem Biophys Res Commun 2012;418:451456.

15 Kulkarni SK, Dhir A: Berberine: a plant alkaloid with therapeutic potential for central nervous system disorders. Phytother Res 2010;24:317-324.

16 Rojsanga P, Gritsanapan W, Suntornsuk L: Determination of berberine content in the stem extracts of Coscinium fenestratum by TLC densitometry. Med Princ Pract 2006;15:373-378.

17 Bova S, Padrini R, Goldman WF, Berman DM, Cargnelli G: On the mechanism of vasodilating action of berberine: possible role of inositol lipid signaling system. J Pharmacol Exp Ther 1992;261:318-323.

18 Akhter MH, Sabir M, Bhide NK: Anti-inflammatory effect of berberine in rats injected locally with cholera toxin. Indian J Med Res 1977;65:133-141.

19 Lee YS, Kim WS, Kim KH, Yoon MJ, Cho HJ, Shen Y, Ye JM, Lee CH, Oh WK, Kim CT, Hohnen-Behrens C, Gosby A, Kraegen EW, James DE, Kim JB: Berberine, a natural plant product, activates AMP-activated protein kinase with beneficial metabolic effects in diabetic and insulin-resistant states. Diabetes 2006;55:22562264.

20 Zhaojie M, Ming Z, Shengnan W, Xiaojia B, Hatch GM, Jingkai G, Li C: Amorphous solid dispersion of berberine with absorption enhancer demonstrates a remarkable hypoglycemic effect via improving its bioavailability. Int J Pharm 2014;467:50-59.

21 Pirillo A, Catapano AL: Berberine, a plant alkaloid with lipid- and glucose-lowering properties: From in vitro evidence to clinical studies. Atherosclerosis 2015;243:449-461.

22 Wu X, Li Q, Xin H, Yu A, Zhong M: Effects of berberine on the blood concentration of cyclosporin A in renal transplanted recipients:clinical and pharmacokinetic study. Eur J Clin Pharmacol 2005;61:567-572.

23 Zhang H, Wei J, Xue R, Wu JD, Zhao W, Wang ZZ, Wang SK, Zhou ZX, Song DQ Wang YM, Pan HN, Kong WJ, Jiang JD: Berberine lowers blood glucose in type 2 diabetes mellitus patients through increasing insulin receptor expression. Metabolism 2010;59:285-292.

24 Pérez-Rubio KG, González-Ortiz M, Martínez-Abundis E, Robles-Cervantes JA, Espinel-Bermúdez MC: Effect of berberine administration on metabolic syndrome, insulin sensitivity, and insulin secretion.Metab Syndr Relat Disord 2013;11:366-369.

25 Meng S, Wang LS, Huang ZQ, Zhou Q, Sun YG, Cao JT, Li YG, Wang CQ: Berberine ameliorates inflammation in patients with acute coronary syndrome following percutaneous coronary intervention. Clin Exp Pharmacol Physiol 2012;39:406-411.

26 Liu WH, Hei ZQ, Nie H, Tang FT, Huang HQ, Li XJ, Deng YH, Chen SR, Guo FF, Huang WG, Chen FY, Liu PQ: Berberine ameliorates renal injury in streptozotocin-induced diabetic rats by suppression of both oxidative stress and aldose reductase. Chin Med J (Engl) 2008;121:706-712.

27 Moghaddam HK, Baluchnejadmojarad T, Roghani M, Khaksari M, Norouzi P, Ahooie M, Mahboobi F: Berberine ameliorate oxidative stress and astrogliosis in the hippocampus of STZ-induced diabetic rats. Mol Neurobiol 2014;49:820-826.

28 Roscioni SS, Heerspink HJ, de Zeeuw D: The effect of RAAS blockade on the progression of diabetic nephropathy. Nat Rev Nephrol 2014;10:77-87.

29 White KE, Bilous RW: Estimation of podocyte number: a comparison of methods. Kidney Int 2004;66:663667.

30 Mifsud SA, Allen TJ, Bertram JF, Hulthen UL, Kelly DJ, Cooper ME, Wilkinson-Berka JL, Gilbert RE: Podocyte foot process broadening in experimental diabetic nephropathy: amelioration with renin-angiotensin blockade. Diabetologia 2001;44:878-882.

31 Katsuya K, Yaoita E, Yoshida Y, Yamamoto Y, Yamamoto T: An improved method for primary culture of rat podocytes. Kidney Int 2006;69:2101-2106.

32 White KE, Bilous RW: Estimation of podocyte number: a comparison of methods. Kidney Int 2004;66:663667.

33 Gaddam K, Pimenta E, Thomas SJ, Cofield SS, Oparil S, Harding SM, Calhoun DA: Spironolactone reduces severity of obstructive sleep apnoea in patients with resistant hypertension: a preliminary report. J Hum Hypertens 2010;24:532-537.

34 Guichard JL, Clark DR, Calhoun DA, Ahmed MI: Aldosterone receptor antagonists: current perspectives and therapies. Vasc Health Risk Manag 2013;9:321-331. 


\section{Cellular Physiology Cell Physiol Biochem 2016;39:217-228 \begin{tabular}{ll|l} 
DOI: 10.1159/000445618 & $\begin{array}{l}\text { O 2016 The Author(s). Published by S. Karger AG, Basel } \\
\text { www.karger.com/cpb }\end{array}$
\end{tabular} \\ Wang et al.: Berberine Improved Aldo-Induced Podocyte Injury}

35 Jeong HW, Hsu KC, Lee JW, Ham M, Huh JY, Shin HJ, Kim WS, Kim JB: Berberine suppresses proinflammatory responses through AMPK activation in macrophages. Am J Physiol Endocrinol Metab 2009;296:E955-E964.

36 Wu N, Sarna LK, Siow YL, O K: Regulation of hepatic cholesterol biosynthesis by berberine during hyperhomocysteinemia. Am J Physiol Regul Integr Comp Physiol 2011;300:R635-R643.

37 Lee TS, Pan CC, Peng CC, Kou YR, Chen CY, Ching LC, Tsai TH, Chen SF, Lyu PC, Shyue SK: Anti-atherogenic effect of berberine on LXRalpha-ABCA1-dependent cholesterol efflux in macrophages. J Cell Biochem 2010;111:104-110.

38 Gu S, Cao B, Sun R, Tang Y, Paletta JL, Wu XL, Liu L, Zha W, Zhao C, Li Y, Ridlon JM, Hylemon PB, Zhou H, Aa J, Wang G: Correction: A metabolomic and pharmacokinetic study on the mechanism underlying the lipidlowering effect of orally administered berberine. Mol Biosyst 2015;11:664.

39 Hu Y, Young AJ, Ehli EA, Nowotny D, Davies PS, Droke EA, Soundy TJ, Davies GE: Metformin and berberine prevent olanzapine-induced weight gain in rats. PLOS One 2014;9:93310.

40 Keidar S, Kaplan M, Pavlotzky E, Coleman R, Hayek T, Hamoud S, Aviram M: Aldosterone administration to mice stimulates macrophage NADPH oxidase and increases atherosclerosis development: a possible role for angiotensin-converting enzyme and the receptors for angiotensin II and aldosterone. Circulation 2004;109:2213-2220.

41 Ogawa Y, Mukoyama M, Yokoi H, Kasahara M, Mori K, Kato Y, Kuwabara T, Imamaki H, Kawanishi T, Koga K, Ishii A, Tokudome T, Kishimoto I, Sugawara A, Nakao K: Natriuretic peptide receptor guanylyl cyclase-A protects podocytes from aldosterone-induced glomerular injury. J Am Soc Nephrol 2012;23:1198-1209.

42 Jean-Marc Zingg, Roberta Ricciarelli, Angelo Azzi: Scavenger Receptors and Modified Lipoproteins: Fatal Attractions? IUBMB Life 2004;49:397-403.

43 Li K, Yao W, Zheng X, Liao K: Berberine promotes the development of atherosclerosis and foam cell formation by inducingscavenger receptor A expression in macrophage. Cell Res 2009;19:1006-1017.

44 Zhang W, Su X, Gao Y, Sun B, Yu Y, Wang X, Zhang F: Berberine protects mesenchymal stem cells against hypoxia-induced apoptosis in vitro. Biol Pharm Bull 2009;32:1335-1342.

45 Song B, Scheuner D, Ron D, Pennathur S, Kaufman RJ: Chop deletion reduces oxidative stress, improves beta cell function, and promotes cell survival in multiple mouse models of diabetes. J Clin Invest 2008;118:3378-3389.

46 Chen Y, Gui D, Chen J, He D, Luo Y, Wang N: Down-regulation of PERK-ATF4-CHOP pathway by Astragaloside IV is associated with the inhibition of endoplasmic reticulum stress-induced podocyte apoptosis in diabetic rats. Cell Physiol Biochem 2014;33:1975-1987.

47 Urra H, Dufey E, Lisbona F, Rojas-Rivera D, Hetz C: When ER stress reaches a dead end. Biochim Biophys Acta 2013;1833:3507-3517. 